\title{
Synthesis of novel bicarbocyclic nucleosides
}

\author{
Vasu Nair* ${ }^{a, b}$ and Geun-Sook Jeon ${ }^{b}$ \\ ${ }^{a}$ Department of Pharmaceutical and Biomedical Sciences, The University of Georgia, \\ Athens, GA 30602 \\ ${ }^{b}$ Department of Chemistry, University of Iowa, Iowa City, IA 52242 \\ E-mail:vnair@rx.uga.edu
}

(received 23 Jul 04; accepted 01 Oct 04; published on the web 08 Oct 04)

\begin{abstract}
The stereospecific syntheses of new bicarbocyclic nucleosides are described. The regioisomers were differentiated by selective INEPT NMR studies and the stereochemistry of these compounds was confirmed by differential NOE NMR experiments. Antiviral results are mentioned.
\end{abstract}

Keywords: Carbocyclic nucleosides, synthesis, NOE, stereochemistry, antiviral

\section{Introduction}

There is increasing interest in nucleosides where the oxygen of the furan ring is replaced by a methylene group. Due to the absence of the glycosidic bond, these compounds referred to as carbocyclic nucleosides are more stable with respect to degradation by enzymes such as phosphorylases. ${ }^{1}$ Isomeric nucleosides where the base is transposed from the 1'- to the 2'-position can also be viewed as carbocyclic nucleosides because the nucleobase is not attached at the glycosidic position. ${ }^{2,3}$ Many carbocyclic nucleosides are known including those where the ribose unit has been replaced by cyclopropane, ${ }^{4}$ cyclobutane, ${ }^{5}$ cyclopentane, ${ }^{6}$ and cyclohexane. ${ }^{7}$ Carbocyclic nucleosides have shown potential as antiviral agents against HIV, HSV and other viruses. ${ }^{8-10}$ Modification of the sugar moiety further to include fused ring systems ${ }^{11}$ has expanded the area of carbocyclic nucleosides. However, bicyclo[2.2.1] carbocyclic nucleosides have not been synthesized or investigated and this is the subject of this communication. Our targeted nucleosides have the secondary hydroxyl group whereas standard nucleosides have the primary hydroxyl group. It has been well known that some biologically active compounds, such as 5'noraristeromycin, ${ }^{12}$ have a secondary hydroxyl group instead of hydroxymethyl group. We wish 
to report on the stereospecific synthesis of novel type of bicyclo[2.2.1] carbocyclic nucleoside analogues.

\section{Results and Discussion}

The synthesis commenced with commercially available 5-norbornen-2-ol (1) which was a mixture of exo and endo isomers. Treatment of $\mathbf{1}$ with tosyl chloride in pyridine gave a mixture of exo- and endo-5-norbornen-2-tosylate (2) in 80\% yield (Scheme 1). The coupling reactions with nucleoside bases, adenine, thymine, and uracil in the presence of $\mathrm{K}_{2} \mathrm{CO}_{3}$ and 18-crown-6 in DMF at $100-110{ }^{\circ} \mathrm{C}$ for 3 days gave only one stereoisomeric product, 2(S)-(nucleobase)-5norbornene (3a-c) in approximately 30\% yield. The relatively low yield of the desired compound can be explained by the formation of the elimination product, 2,5-norbornadiene. The stereochemistry of compound 3 was confirmed by differential NOE experiments. For example, in case of 3a, irradiation of $\mathrm{H}-8$ of the adenine base resulted in the enhancement of the signal for $\mathrm{H}$ 7'. The chemical shift of the latter was assigned by homodecoupling experiments. The NOE data confirmed the proximity of the adenine base to the bridgehead position and that the nucleophilic displacement of the tosyl group by the adenine base had occurred from the exo face.

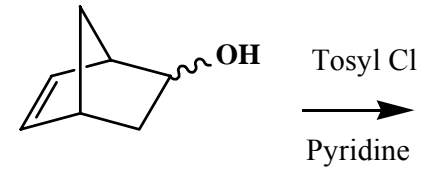

1

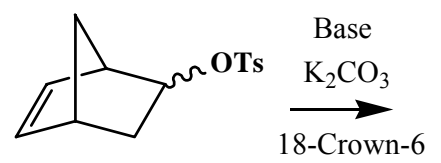

2

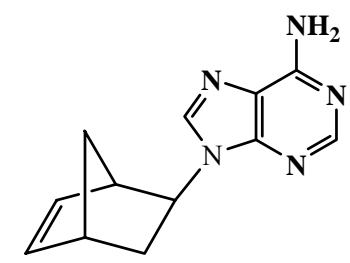

3a

$$
\begin{aligned}
& \text { 3b, Base }=T \\
& \text { 3c, Base }=U
\end{aligned}
$$

(1) $\mathrm{BH}_{3}, \mathrm{THF}$

(2) $\mathrm{H}_{2} \mathrm{O}_{2}, \mathrm{NaOH}$

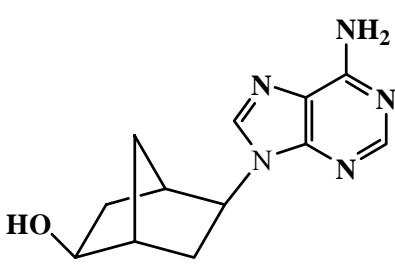

5a (Minor)

$5 \mathbf{b}$, Base $=\mathrm{T}$

5c, Base $=\mathrm{U}$

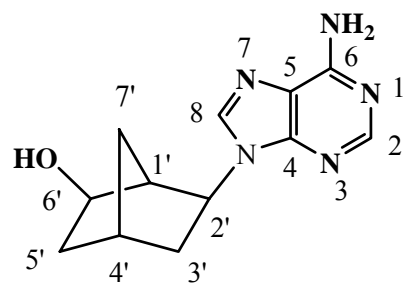

4a (Major)

4b, Base $=\mathrm{T}$

4c, Base $=\mathrm{U}$

\section{Scheme 1}


Hydroboration of 3 was accomplished by using 4 equivalents of $\mathrm{BH}_{3}-\mathrm{THF}$ in THF, followed by oxidation with $30 \% \mathrm{H}_{2} \mathrm{O}_{2}$ and $2 \mathrm{~N} \mathrm{NaOH}(\mathrm{aq})$ to afford two regioisomers in $80 \%$ yield: norbornan-6(R)-ol (4) as a major product (45\%) and norbornan-5(S)-ol (5) as a minor product (35\%) (Scheme 1). These regioisomers were separated by reversed-phase HPLC on Amberlite $\mathrm{XAD}-4$ resin as the stationary phase with ethanol/water as the eluting solvent.

The cytidine derivative (4d) was synthesized from uridine $\mathbf{3 c}$ which was treated first with a solution of phosphorus oxychloride and 1,2,4-triazole in pyridine, followed by treatment with $\mathrm{NH}_{4} \mathrm{OH}$ to afford 2(S)-[4-amino-2-oxo-1(2H)-pyrimidinyl]-5-norbornene (3d). The cytidine derivative 3d was treated with 4 mol equivalents of $\mathrm{BH}_{3}$-THF followed by $30 \% \mathrm{H}_{2} \mathrm{O}_{2}$ and $\mathrm{NaOH}$ to afford 2(S)-[4-amino-2-oxo-1(2H)-pyrimidinyl]-norbornan-6 ( $R$ )-ol (4d) in 40\% yield and 2(S)-[4-amino-2-oxo-1(2H)-pyrimidinyl]-norbornan-5(S)-ol (5d) in 30\% (Scheme 2).

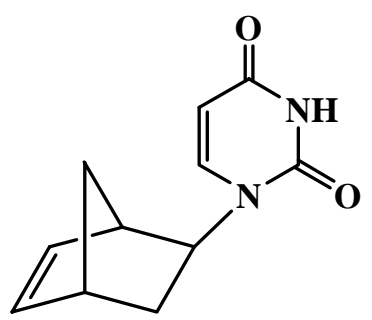

3c

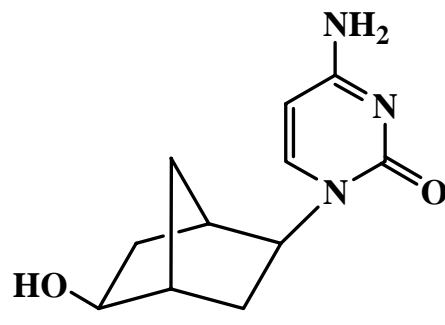

5d
(1) $\mathrm{POCl}_{3}$, Triazole

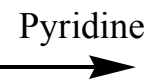

(2) $\mathrm{NH}_{4} \mathrm{OH}$

$3 d$

(1) $\mathrm{BH}_{3}, \mathrm{THF}$

(2) $\mathrm{H}_{2} \mathrm{O}_{2}, \mathrm{NaOH}$

$+$

\section{Scheme 2}

The structures of 4 and 5 were confirmed by ${ }^{1} \mathrm{H}$ and ${ }^{13} \mathrm{C}$ NMR data and additional NMR experiments such as homodecoupling, DEPT, and NOE. The two regioisomers were differentiated by selective INEPT ${ }^{1} \mathrm{H}-{ }^{13} \mathrm{C}$ correlations. ${ }^{13}$ For example, in the case of $4 a$, selective irradiation of the endo-H-2' resulted in the enhancement of the resonances at $33.1\left(\mathrm{C}-7^{\prime}\right), 36.1$ (C-4'), $38.70\left(\mathrm{C}-3^{\prime}\right), 51.2\left(\mathrm{C}-1^{\prime}\right), 73.0\left(\mathrm{C}-6^{\prime}\right), 139.6(\mathrm{C}-8)$, and 150.2 (C-4). No enhancement was observed at $40.9 \mathrm{ppm}\left(\mathrm{C}-5^{\prime}\right)$. Enhancement of the resonance at $72.7 \mathrm{ppm}$ indicates that the 
hydroxyl group is attached at the C-6' position. The exo-addition of the hydroxyl group to the double bond was confirmed by NOE experiments. For example, in the case of $\mathbf{4 a}$, irradiation of H-6' resulted in enhancement of $\mathrm{H}-2^{\prime}$ by $15 \%$ (Figure 1).

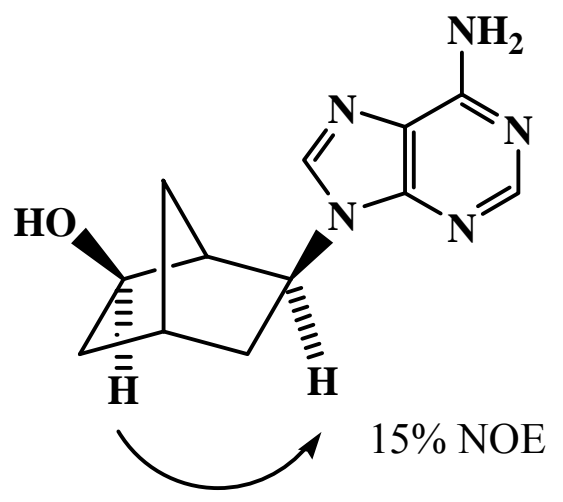

4a

Figure 1. Differential NOE Experiments with 4a.

In summary, the hitherto unknown bicyclo [2.2.1] carbocyclic nucleosides have been prepared. These compounds maintain the 1,3-cis- relationship between the nucloside base and the hydroxyl group. In vitro anti-HIV studies showed that the target compounds were inactive. Further antiviral studies are in progress and will be reported elsewhere.

\section{Experimental Section}

General Procedures. Melting points reported were uncorrected and were determined on an Electrothermal Engineering Ltd. melting point apparatus. Ultraviolet (UV) spectra were recorded on a Cary 3 UV-Visible spectrophotometer. ${ }^{1} \mathrm{H}$ NMR and ${ }^{13} \mathrm{C}$ NMR were recorded on a AC-300 and WM-360 instruments. Chemical shifts are referenced to an internal TMS standard for $1 \mathrm{H}-$ NMR spectra and to solvent $\left(\mathrm{CDCl}_{3}\right.$, DMSO- $\mathrm{d}_{6}$, Acetone- $\mathrm{d}_{6}$ or $\left.\mathrm{CD}_{3} \mathrm{OD}\right)$ for ${ }^{13} \mathrm{C}$ NMR spectra. Column chromatographic separations were carried out using 230-400 mesh silica gel. HPLC separations were carried out at 80 psi on Altex columns packed with Amberlite XAD-4 resin. Fractions were monitored by a Pharmacia UV-2 ultraviolet monitor and were collected on a Gilson FC-100 fraction collector. Purities of intermediates and final products were determined by a combination of ${ }^{1} \mathrm{H}$ and ${ }^{13} \mathrm{C}$ NMR spectra, quantitative UV data and HPLC analysis.

2-(S)-[6-Amino-9(H)-purin-9-yl]]-5-norbornene (3a). To a solution of 2 (5.2g, $19.67 \mathrm{mmole})$ in DMF $(100 \mathrm{~mL})$ were added adenine $(4.0 \mathrm{~g}, 29.5 \mathrm{mmole}), \mathrm{K}_{2} \mathrm{CO}_{3}(4.35 \mathrm{~g}, 31.47 \mathrm{mmole})$ and 18-crown-6 (6.76 g, 25.57 mmole). The resulting solution was warmed to $100-105{ }^{\circ} \mathrm{C}$ for $48 \mathrm{~h}$. 
The solvent was removed in under high vacuum and the residue was purified on silica gel with $10-15 \%$ methanol/chloroform to give only one isomer 3 a $(1.34 \mathrm{~g}, 5.9 \mathrm{mmol}, 30 \%$ yield, $39 \%$ conversion). Starting material $(1.2 \mathrm{~g}, 4.54 \mathrm{mmol}, 30 \%)$ was recovered together with 2,5norbornadiene. ${ }^{1} \mathrm{H}$ NMR $\left(\mathrm{CDCl}_{3}\right) \delta 1.26\left(\mathrm{~s}, 1 \mathrm{H}, \mathrm{H}-7^{\prime}\right), 1.77\left(\mathrm{~s}, 1 \mathrm{H}, \mathrm{H}-7^{\prime}\right), 1.94\left(\mathrm{dt}, 1 \mathrm{H}, \mathrm{H}-3^{\prime}\right.$, $\mathrm{J}=3.68,7.35 \mathrm{~Hz}$ ), 2.03 (m, 1H, H-3'), 3.11 (s, 1H, H-4'), 3.19 (s, 1H, H-1'), 4.51 (dd, 1H, H-2', $\mathrm{J}=3.88,7.97), 6.26$ (dd, 1H, H-6', J=3.17, 5.69 Hz), 6.32 (dd, 1H, H-5', J=2.91, 5.89 Hz), 6.34 (s, 2H, $\mathrm{NH}_{2}$, exchangeable), 7.97 (s, 1H, H-8), 8.37 (s, 1H, H-2); ${ }^{13} \mathrm{C}$ NMR $\delta 33.3$ (C-7'), 41.4 (C4'), 46.4 (C-3'), 47.7 (C-1'), 54.9 (C-2'), 119.9 (C-5), 134.4 (C-6'), 137.9 (C-8), 139.7 (C-5'), 150.5 (C-4), 152.7 (C-2), 155.7 (C-6); UV $\lambda_{\max }(\mathrm{MeOH}) 260 \mathrm{~nm}$.

2(S)-[6-Amino-9(H)-purin-9-yl-norbornan-6(R)-ol (4a) and 2(S)-[6-amino-9(H)-purin-9-yl]norbornan-5(S)-ol (5a). To a solution of 3a $(0.55 \mathrm{~g}, 2.4 \mathrm{mmole})$ in THF $(25 \mathrm{~mL})$ was added $1.0 \mathrm{M} \mathrm{BH}_{3} \cdot \mathrm{THF}(9.7 \mathrm{~mL}, 9.7 \mathrm{mmole})$ at $0{ }^{\circ} \mathrm{C}$. The resulting solution was warmed to room temperature and stirred for $5 \mathrm{~h}$. It was quenched with water, followed by treatment with $30 \%$ $\mathrm{H}_{2} \mathrm{O}_{2}$ and $2 \mathrm{~N} \mathrm{NaOH}$ (aq). It was neutralized with dil. $\mathrm{HCl}$. The solvent was removed under reduced pressure and then the residue was purified on silica gel with $15-20 \%$ methanol/chloroform to give a mixture of two isomers, $4 \mathbf{a}$ and $\mathbf{5 a}(0.5 \mathrm{~g}, 2.039 \mathrm{mmol}, 80 \%)$. The two regioisomers were separated by reversed-phase HPLC with water-ethanol as the eluting solvent. Spectral data for 4a (45\%): mp 199-201 ${ }^{\circ} \mathrm{C},{ }^{1} \mathrm{H}$ NMR $\left(\mathrm{CD}_{3} \mathrm{OD}\right) \delta 1.46\left(\mathrm{~m}, 1 \mathrm{H}, \mathrm{H}-5^{\prime}\right)$, 1.69 (d, 1H, H-5', J=10.57), 1.85 (m, 3H, H-7', H-3'), 2.01 (m, 1H, H-3'), 2.50 (br s, 1H, H-4'), 2.54 (br s, 1H, H-1'), 4.04 (d, 1H, H-6', J=6.59 Hz), 4.40 (dd, 1H, H-2', J=4.79, 8.24 Hz), 8.21 (s, 1H, H-8), 8.22 (s, 1H, H-2); ${ }^{13} \mathrm{C}$ NMR $\delta 33,1$ (C-7'), 36.1 (C-4'), 38.7 (C-3'), 40.9 (C-5'), 51.2 (C-1'), 55.0 (C-2'), 73.0 (C-6'), 120.1 (C-5), 139.6 (C-8), 150.2 (C-4), 153.2 (C-2), 157.0 (C-6; UV $\lambda_{\max }\left(\mathrm{H}_{2} \mathrm{O}\right) 260 \mathrm{~nm}\left(\varepsilon\right.$ 13,500): Spectral data for 5a (35\%): mp 125-126 ${ }^{\circ} \mathrm{C},{ }^{1} \mathrm{H}$ NMR $\left(\mathrm{CD}_{3} \mathrm{OD}\right) \delta 1.53$ (m, 1H, H-6'), 1.62 (d, 1H, H-6', J=11.92 HZ), 1.89 (m, 4H, H-7', H-3'), 2.42 (d, 1H, H-4', J=4.14 Hz), 2.63 (d, 1H, H-1', J=4.65 Hz), 3.89 (d, 1H, H-5', J=6.38 Hz), 4.39 (m, 1H, H-2'), 8.04 (s, 1H, H-8), 8.25 (s, 1H, H-2); ${ }^{13} \mathrm{C}$ NMR $\delta 32.8$ (C-7'), 33.9 (C-4'), 39.7 (C-3'), 42.1 (C-6'), 44.6 (C-1'), 57.6 (C-2'), 73.1 (C-5'), 119.9 (C-5), 138.4 (C-8), 149.9 (C-4), 152.7 (C-2), 156.1 (C-6); UV $\lambda_{\max }\left(\mathrm{H}_{2} \mathrm{O}\right), 260 \mathrm{~nm}(\varepsilon 11,400)$.

2(S-)-[3,4-Dihydro-2,4-dioxo-5-methyl-1(2H)-pyrimidinyl]-5-norbornene (3b). To a solution of $2(2.94 \mathrm{~g}, 11.12 \mathrm{mmole})$ in DMF $(100 \mathrm{~mL})$ were added thymine $(2.10 \mathrm{~g}, 16.68 \mathrm{mmole})$, $\mathrm{K}_{2} \mathrm{CO}_{3}(2.46 \mathrm{~g}, 17.79 \mathrm{mmol})$, and 18 -crown-6 $(3.82 \mathrm{~g}, 14.46 \mathrm{mmol})$. The resulting solution was warmed to $110^{\circ} \mathrm{C}$ for $48 \mathrm{~h}$. The solvent was removed under vacuum and the residue was purified on silica gel with $5-10 \%$ methanol/chloroform to give an $O$-coupled isomer $(0.2 \mathrm{~g}, 0.916 \mathrm{mmol}$, $8.3 \%)$ and the desired $N$-coupled isomer $3 \mathbf{b}(0.26 \mathrm{~g}, 1.19 \mathrm{mmol}, 10.7 \%)$. Spectral data for $3 \mathbf{b}:{ }^{1} \mathrm{H}$ NMR $\left(\mathrm{CDCl}_{3}\right) \delta 1.46\left(\mathrm{~m}, 1 \mathrm{H}, \mathrm{H}-7^{\prime}\right), 1.58\left(\mathrm{~m}, 1 \mathrm{H}, \mathrm{H}-7^{\prime}\right), 1.73\left(\mathrm{~m}, 1 \mathrm{H}, \mathrm{H}-3^{\prime}\right), 1.95$ (m, 4H, H-3', $\mathrm{CH}_{3}$ ), 3.00 (m, 1H, H-4'), 4.22 (dd, 1H, H-1', J=3.07, 7.07), 6.16 (dd, 1H, H-6', J=3.29, $5.53 \mathrm{~Hz}$ ), 6.28 (dd, 1H, H-5', J=2.80, $4.64 \mathrm{~Hz}$ ), 7.23 (s, 1H, H-6), 9.97 (br s, $1 \mathrm{H}, \mathrm{NH}$, exchangeable); ${ }^{13} \mathrm{C}$ NMR $\delta 12.5\left(\mathrm{CH}_{3}\right), 33.6\left(\mathrm{C}-7^{\prime}\right), 40.9\left(\mathrm{C}-4^{\prime}\right), 45.3\left(\mathrm{C}-1^{\prime}\right), 46.1\left(\mathrm{C}-3^{\prime}\right), 56.8\left(\mathrm{C}-2^{\prime}\right), 106.6(\mathrm{C}-5)$, 134.3 (C-6'), 136.2 (C-6), 140.1 (C-5'), 151.8 (C-2), 164.2 (C-4); UV $\lambda_{\max }(\mathrm{MeOH}) 271 \mathrm{~nm}$. 
2(S)-[3, 4-Dihydro-2, 4-dioxo-5-methyl-1(2H)-pyrimidinyl]-norbornan-6 $(R)-$ ol $(4 b)$ and 2(S)-[3,4-dihydro-2,4-dioxo-5-methyl-1(2H)-pyrimidinyl]-norbornan-5(S)-ol (5b). To a solution of $3 \mathbf{b}(0.25 \mathrm{~g}, 1.15 \mathrm{mmole})$ in THF $(20 \mathrm{~mL})$ was added $1.0 \mathrm{M} \mathrm{BH} \mathrm{BH}_{3}$ THF $(2.86 \mathrm{ml}$, $2.86 \mathrm{mmol}$ ) at $0^{\circ} \mathrm{C}$. The resulting solution was warmed to room temperature and stirred for $5 \mathrm{~h}$ and worked up as described for $\mathbf{4 a}$ and $\mathbf{5 a}$ to give the regioisomers, $\mathbf{4 b}$ and $\mathbf{5 b}$ (70 $\mathrm{mg}$, $0.296 \mathrm{mmol}, 26 \%)$. The two isomers were separated by reversed-phase HPLC with water-ethanol as the eluting solvent. Data for $\mathbf{4 b}$ : $\mathrm{mp} 98-100{ }^{\circ} \mathrm{C}$ (lyophilized powder); $1 \mathrm{H} \mathrm{NMR}\left(\mathrm{CDCl}_{3}\right) \delta$ 1.41 (m, 2H, H-5'), 1.75 (m, 3H, H-7', H-3'), 1.90 (m, 4H, H-3', $\mathrm{CH}_{3}$ ), 2.33 (s, 1H, H-4'), 2.46 (s, 1H, H-1'), 3.99 (d, 1H, H-6', J=16.33 Hz), 4.20 (m, 1H, H-2'), 7.15 (s, 1H, H-6), 8.77 (br s, 1H, $\mathrm{NH}$, exchangeable); ${ }^{13} \mathrm{C}$ NMR $\delta 13.0\left(\mathrm{CH}_{3}\right), 33.2\left(\mathrm{C}-7^{\prime}\right), 35.0\left(\mathrm{C}-4^{\prime}\right), 38.4\left(\mathrm{C}-3^{\prime}\right), 40.4\left(\mathrm{C}-5^{\prime}\right)$, 48.9 (C-1'), 55.0 (C-2'), 73.1 (C-6'), 111.0 (C-5), 136.2 (C-6), 151.4 (C-2), 163.5 (C-4); UV $\lambda_{\max }$ $\left(\mathrm{H}_{2} \mathrm{O}\right) 272 \mathrm{~nm}(\epsilon 5,600)$. Data for 5b: mp $115-117^{\circ} \mathrm{C}$ (lyophilized powder); ${ }^{1} \mathrm{H}$ NMR $\left(\mathrm{CDCl}_{3}\right) \delta$ 1.46 (m, 3H, H-6', H-7'), 1.89 (m, 6H, H-3', H-7', CH 3 ), 2.33 (d, 1H, H-4', J=4.57 Hz), 2.47 (d, 1H, H-1', J=4.89 Hz), 3.91 (m, 1H, H-5'), 4.12 (m, 1H, H-2'), 7.18 (s, 1H, H-6), 8.31 (br s, 1H, $\mathrm{NH}$, exchangeable); ${ }^{13} \mathrm{C}$ NMR $\delta 12.7\left(\mathrm{CH}_{3}\right), 32.9\left(\mathrm{C}-7^{\prime}\right), 34.7\left(\mathrm{C}-4^{\prime}\right), 39.7\left(\mathrm{C}-3^{\prime}\right), 40.2\left(\mathrm{C}-6^{\prime}\right)$, 44.0 (C-1'), 58.3 (C-2'), 73.2 (C-5'), 110.1 (C-5), 136.1 (C-6), 151.1 (C-2), 163.0 (C-4).

2(S)-[3,4-Dihydro2,-4-dioxo-1 (2H)-pyrimidinyl]-norbornan-6 $(R)$-ol $(4 c)$ and 2(S)-[3,4dihydro-2, 4-dioxo-1(2H)-pyrimidinyl]-norbornan-5(S)-ol (5c). Intermediate 3c was prepared as described for $3 \mathbf{b}$ (yield: $56 \%):{ }^{1} \mathrm{H}$ NMR $\left(\mathrm{CDCl}_{3}\right) \delta 1.45\left(\mathrm{dt}, 1 \mathrm{H}, \mathrm{H}-3^{\prime}, \mathrm{J}=3.62 \mathrm{~Hz}\right), 1.57(\mathrm{~d}, 1 \mathrm{H}$, H-7', J=9.09 Hz), 1.74 (d, 1H, H-7', J=8.04 Hz), 2.00 (m, 1H, H-3'), 3.00 (m, 2H, H-1', H-4'), 4.22 (m, 1H, H-2'), 5.73 (d, 1H, H-5, J=8.11 Hz), 6.17 (dd, 1H, H-6', J=3.36, $5.58 \mathrm{~Hz}$ ), 6.30 (dd, $1 \mathrm{H}, \mathrm{H}-5^{\prime}, \mathrm{J}=2.84,5.63 \mathrm{~Hz}$ ), 7.42 (d, $\left.1 \mathrm{H}, \mathrm{H}-6, \mathrm{~J}=7.94 \mathrm{~Hz}\right), 9.76$ (br s, $1 \mathrm{H}, \mathrm{NH}$, exchangeable); ${ }^{13} \mathrm{C}$ NMR $\delta 34.1$ (C-7'), 41.2 (C-4'), 45.3 (C-1'), 46.2 (C-3'), 52.3 (C-2'), 101.4 (C-5), 134.2 (C-6'), 140.5 (C-6), 141.6 (C-5'), 151.7 (C-2), 163.6 (C-4). Compound 3c was converted to 4c and 5c $(25 \%)$ as described for $\mathbf{4 b}$ and $\mathbf{5 b}$. Data for $\mathbf{4 c}{ }^{1} \mathrm{H}$ NMR $\left.\mathrm{CD}_{3} \mathrm{OD}\right) \delta 1.42\left(\mathrm{~m}, 3 \mathrm{H}, \mathrm{H}-5^{\prime}, \mathrm{H}-7^{\prime}\right)$, 1.78 (m, 2H, H-7', H-3'), 1.91 (m, 1H, H-3'), 2.31 (s, 1H, H-4'), 2.45 (s, 1H, H-1'), 3.80 (m, 1H, H-6'), 4.14 (dd, 1H, H-2', J=4.79, 5.11), 5.69 (d, 1H, H-5, J=8.07 Hz), 7.45 (d, 1H, H-6, J=8.12 $\mathrm{Hz}) ;{ }^{13} \mathrm{C}$ NMR $\left(\mathrm{CD}_{3} \mathrm{OD}\right) \delta 33.4\left(\mathrm{C}-7^{\prime}\right), 35.4\left(\mathrm{C}-4^{\prime}\right), 39.0\left(\mathrm{C}-3^{\prime}\right), 43.8\left(\mathrm{C}-5^{\prime}\right), 56.0\left(\mathrm{C}-2^{\prime}\right), 72.5(\mathrm{C}-$ 6'), 101.8 (C-5), 141.0 (C-6), 151.5 (C-2), 164.3 (C-4).

2(S)-[4-Amino-2-oxo-1(2H)-pyrimidinyl]-5-norbornene (3d). To a solution of 3c $(0.18 \mathrm{~g}$, $0.88 \mathrm{mmole})$ in pyridine $(7 \mathrm{~mL})$ was added phosphorus oxychloride $(0.16 \mathrm{ml}, 1.76 \mathrm{mmol})$ and $1,2,4$-triazole $(0.24 \mathrm{~g}, 3.52 \mathrm{~mol})$ in pyridine $(7 \mathrm{~mL})$ at $0{ }^{\circ} \mathrm{C}$. The resulting solution was warmed to room temperature and stirred for $26 \mathrm{~h}$, followed by treatment with $\mathrm{NH}_{4} \mathrm{OH}(2 \mathrm{ml}, 17.6$ mmole $)$ and further stirring for $12 \mathrm{~h}$. The solvent was removed and the residue was purified on silica gel with $10-15 \%$ methanol/chloroform to give $3 d$ (60 $\mathrm{mg}, 0.295 \mathrm{mmol}, 34 \%$ yield). ${ }^{1} \mathrm{H}$ NMR $\left(\mathrm{CD}_{3} \mathrm{OD}\right) \delta 1.44\left(\mathrm{dt}, 1 \mathrm{H}, \mathrm{H}-3^{\prime}, \mathrm{J}=3.59\right), 1.64\left(\mathrm{~m}, 2 \mathrm{H}, \mathrm{H}-7^{\prime}\right), 1.94\left(\mathrm{~m}, 1 \mathrm{H}, \mathrm{H}-3^{\prime}\right), 2.96$ (s, 1H, H4'), 3.01 (s, 1H, H-1'), 4.14 (m, 1H, H-2'), 5.87 (d. 1H, H-5, J=7.39 Hz), 6.18 (dd, 1H, H-6', $\mathrm{J}=3.33,5.62 \mathrm{~Hz}), 6.32\left(\mathrm{dd}, 1 \mathrm{H}, \mathrm{H}-5^{\prime}, \mathrm{J}=2.85,5.55 \mathrm{~Hz}\right), 7.65(\mathrm{~d}, 1 \mathrm{H}, \mathrm{H}-6, \mathrm{~J}=7.47) ;{ }^{13} \mathrm{C}$ NMR $\left(\mathrm{CD}_{3} \mathrm{OD}\right) \delta 35.3\left(\mathrm{C}-7^{\prime}\right), 42.6\left(\mathrm{C}-4^{\prime}\right), 46.6\left(\mathrm{C}-1^{\prime}\right), 46.9$ (C-3'), 59.3 (C-2'), 95.3 (C-5), 135.7 (C$\left.6^{\prime}\right), 141.5$ (C-5'), 143.3 (C-6), $157.3(\mathrm{C}-2), 164.3(\mathrm{C}-4)$; UV $\lambda_{\max }\left(\mathrm{H}_{2} \mathrm{O}\right) 276.5 \mathrm{~nm}$. 
2(S)-[4-Amino-2-oxo-1(2H)-pyrimidinyl]-norbornan-6(R)-ol (4d) and 2(S)-[4-Amino-2-oxo1(2H)-pyrimidinyl]-norbornan-5(S)-ol (5d). Compounds 4d (31\%) and 5d (6\%) were prepared by hydroboration followed by oxidation as described for 4a and 5a. Data for 4d: $1 \mathrm{H} \mathrm{NMR}$ (CD $\left.{ }_{3} \mathrm{OD}\right) 1.34$ (m, 3H, H-5', H-7'), 1.64 (m, 2H, H-7', H-3'), 1.78 (m, 1H, H-3'), 2.20 (s, 1H, H4'), 2.32 (s, 1H, H-1'), 3.77 (d, 1H, H-6', J=6.34 Hz), 4.08 (m, 1H, H-2'), 5.79 (d, 1H, H-5, $\mathrm{J}=7.42 \mathrm{~Hz}), 7.62$ (d, 1H, H-6, J=7.49 Hz), ${ }^{13} \mathrm{C}$ NMR $\left(\mathrm{CD}_{3} \mathrm{OD}\right) 30.7\left(\mathrm{C}-7\right.$ '), $37.1\left(\mathrm{C}-4^{\prime}\right), 36.8(\mathrm{C}-$ 3'), $38.4\left(\mathrm{C}-5^{\prime}\right), 46.8\left(\mathrm{C}-1^{\prime}\right), 53.8$ (C-2'), 70.2 (C-6'), 91.9 (C-5), 140.2 (C-6), 152.7 (C-2), 163.1 (C-4); UV $\lambda_{\max }\left(\mathrm{H}_{2} \mathrm{O}\right) 275 \mathrm{~nm}(\varepsilon 6,900)$.

\section{Acknowledgements}

Support of this research by National Institutes of Health (NIAID) is gratefully acknowledged.

\section{References}

1. Marquez, V. Advances in Antiviral Drug Design 1996, 2, 89.

2. Nair, V.; Jahnke, T. S. Antimicrob. Agents Chemother. 1995, 39, 1017.

3. Nair, V. In Recent Advances in Nucleosides: Chemistry and Chemotherapy; Chu, C. K., Ed.; Elsevier Science: Amsterdam, Netherlands, 2002, 149.

4. Csuk, R.; Scholz, Y. Tetrahedron 1994, 50, 10431.

5. Maruyama, T,; Hanai, Y.; Sato, Y.; Snoeck, R.; Andrei, G.; Hosoya, M.; Balzarini, J.; DeClercq, E. Chem. Pharm. Bull. 1993, 41, 516.

6. Agrofoglio, L; Suhas, E.; Farese, A.; Condom, R.; Challand, S.R.; Earl, R.A.; Guedj, R. Tetrahedron 1994, 50, 10611.

7. Pérez- Pérez, M-J.; Rozenski, J.; Bussan, R.; Herdewijn, P. J. Org. Chem. 1995, 60, 1531.

8. De Clercq, E. J. Med. Chem. 1995, 38, 2491.

9. De Clerq, E. Nature Rev. Drug Discovery 2002, 11, 13.

10. (a) Howell, H.; Brodfuehrer, P.; Brundidge, S.; Benigni, D.; Saping, C. J. Org. Chem. 1988, 53, 85. (b) De Clercq, E.; Balzarini, J.; Bernaerts, R.; Herdewijn, P.; Verbrüggen, A. Biochem. Biophys. Res. Commun. 1985, 126, 397. (c) Coates, S.; Ingall, H.; Pearson, B.; Penn, C.; Storer, R.; Williamson, R.; Cameron, J. Antiviral Res. 1991, 161. (d) Yaginuma, S.; Muto, N.; Tsujino, M.; Sudate, Y.; Hayashi, M.; Ohani, M. J. Antibiotic. 1981, 34, 359.

11. (a) Chao, Q.; Nair, V. Tetrahedron 1997, 53, 1957. (b) Rodriguez, J. B.; Marquez,V.E.; Nicklau, M.C.; Mitsuya, H.; Barchi, J.J., Jr. J. Med. Chem. 1994, 37, 3389. (c) Rodriguez, J.B.; Marquez, V.E.; Nicklaus, M.C.; Barchi, J.J., Jr. Tetrahedron Lett. 1993, 37, 6233. (d) Chang, H.S.; Bergmeiier, S.C.; Frick, J.A.; Bathe, A.; Rapoport, H. J. Org. Chem. 1994, 59, 5336. 
12. (a) Ghosh, A.; Ritter, A.R.; Miller, J.J. J. Org. Chem. 1995, 60, 5808. (b) Patil, S.D.; Schneller, S.W.; Hosoya, M.; Snoeck, R.; Andrei G.; Balzarini, J.; De Clercq, E. J. Med. Chem. 1992, 35, 3372. (c) Siddiqui, S.M., Oertel, F.P.; Chen, X.; Schneller, S.W. J. Chem. Soc., Chem. Commun. 1993, 708.

13. Nair, V.; Nuesca, Z. J. Am. Chem. Soc. 1992, 114, 7951. 